\title{
System with classical and quantum subsystems in tomographic probability representation
}

\author{
V. N. Chernega ${ }^{\dagger}$, V. I. Man'ko ${ }^{\dagger}$ \\ $\dagger$ P.N. Lebedev Physical Institute, Russian Academy of Sciences \\ Leninskii Prospect, 53, Moscow 119991, Russia \\ Emails: vchernega@gmail.com, manko@sci.lebedev.ru
}

September 27, 2017

\begin{abstract}
Description of system containing classical and quantum subsystems by means of tomographic probability distributions is considered. Evolution equation of the system states is studied.
\end{abstract}

\section{Introduction}

States of a classical particle moving in an environment are described by a probability density $f(q, p)$ on the particle phase-space where position $q$ and momentum $p$ fluctuate. Pure states of a quantum particle are associated with complex wave function $\psi(x)$ [1]. For the quantum particle in an environment the states are identified with the Hermitian nonnegative density operator $\hat{\rho}$ (or its density matrix, e.g., in position representation $\rho\left(x, x^{\prime}\right)$ which is the complex function of two real variables [2, 3. Recently, the tomographic probability representation of both quantum [4, 5] and classical [6, 7] states was introduced. In this representation the classical particle states and the quantum particle states are identified with tomographic probability distribution $w(X, \mu, \nu)$ (called symplectic tomogram) or $w(X, \theta)$ (called optical tomogram) where the random variable $X$ is the particle position measured in the corresponding reference frame of the particle phase-space. The reference frame is labeled by the two real parameters $\mu$ and $\nu$ for symplectic tomogram and by the rotation angle $\theta$ for the optical tomogram. The rotation angle $\theta$ of the reference frame axes in the phase-space is called the local oscillator phase [8] in the cases where the photon quantum states are considered and their Wigner functions are reconstructed [9] using experimental results providing the optical tomogram $w(X, \theta)$ (see, e.g. [10]). In [11] it was pointed out that since the classical and quantum particle states are identified with the same tomographic probability distribution, e.g. symplectic tomogram there exists a possibility to construct the quantum and classical mechanics in a framework of a unified scheme namely using the tomographic probability representation. This idea to suggest a scheme where both classical and quantum mechanics are unified was discussed in the literature before see, e.g. [12]. Recently Elze with collaborators [13] suggested the description of both classical and quantum linear dynamics using phase-space representation of the particle and path integral formalism. The problems of such unified construction of the mechanics which combines both classical and quantum states are related to difference of the states description in classical and quantum domains. The Wigner function $W(q, p)[14$ is similar to classical probability density $f(q, p)$ but nevertheless it is not nonnegative 
probability distribution of measurable particle position and momentum. The aim of this work is to use the particle state description both in classical and quantum domains by the tomographic probability distribution and to suggest the description of the states of systems with classical and quantum subsystems by the joint tomographic probability distributions depending on random classical and quantum positions and to propose evolution equation for the tomograms of such system states compatible with Liouville and von Neumann kinetic equations in classical and quantum domains, respectively.

The paper is organized as follows. In Sec.2 the property of a joint probability distributions $w\left(X_{1}, X_{2}, \theta_{1}, \theta_{2}\right)$ of two random variables $X_{1}$ and $X_{2}$ depending on two extra real parameters $\theta_{1}$ and $\theta_{2}$ and their relation to optical tomograms of a system with two particles are studied. In Sec.3 the joint tomographic probability for a system with classical and quantum subsystems is introduced. In Sec.4 the evolution equation for the tomogram of hybrid system with classical and quantum subsystems is proposed on the base of evolution equation for the optical tomograms of the separated classical and quantum particles found in [15, 16]. In Sec.5 conclusions and perspectives are discussed and entanglement properties of hybrid systems are shortly considered.

\section{Correlations of random variables}

In this Section we study optical tomographic joint probability distribution depending on two random variables. We associate the probability distribution with state of system containing two subsystems. Given a joint probability distributions $w\left(X_{1}, X_{2}, \theta_{1}, \theta_{2}\right)$ of two real random variables $X_{1}$ and $X_{2}$ and two angles $\theta_{1}, \theta_{2}$. The random variables are positions of two particles measured in reference frame in phase space with rotated axes, rotation angles being labeled by $\theta_{1}$ and $\theta_{2}$. The joint probability distribution called optical tomogram satisfies the following conditions of positivity

$$
w\left(X_{1}, X_{2}, \theta_{1}, \theta_{2}\right) \geq 0,
$$

and normalization

$$
\int w\left(X_{1}, X_{2}, \theta_{1}, \theta_{2}\right) d X_{1} d X_{2}=1 \text {. }
$$

There exist following possibilities. If the two particles are classical ones the joint probability distributions describing a state of the particle obeys the positivity condition of the following integral which is the Radon integral transform connecting the tomogram with the probability density describing the system state

$$
\begin{aligned}
& \frac{1}{4 \pi^{2}} \int_{0}^{\pi} \int_{0}^{\pi} d \theta_{1} d \theta_{2} \int_{-\infty}^{+\infty} d \eta_{1} d \eta_{2} d X_{1} d X_{2} w\left(X_{1}, X_{2}, \theta_{1}, \theta_{2}\right)\left|\eta_{1} \eta_{2}\right| \\
& \times \exp i\left[\eta_{1}\left(X_{1}-q_{1} \cos \theta_{1}-p_{1} \sin \theta_{1}\right)+\eta_{2}\left(X_{2}-q_{2} \cos \theta_{2}-p_{2} \sin \theta_{2}\right)\right]=f\left(q_{1}, p_{1}, q_{2}, p_{2}\right) \geq 0 .
\end{aligned}
$$

The function $f\left(q_{1}, p_{1}, q_{2}, p_{2}\right)$ is the probability density of two particles on their phase-space. This function is normalized

$$
\int f\left(q_{1}, p_{1}, q_{2}, p_{2}\right) d q_{1} d q_{2} d p_{1} d p_{2}=1
$$

if the conditional (2) holds. If the tomogram describes a state of two quantum particles it must satisfy the positivity condition for the density operator given by the following integral which is quantum version 
of Radon transform

$$
\begin{aligned}
& \hat{\rho}(1,2)=\frac{1}{4 \pi^{2}} \int_{0}^{\pi} \int_{0}^{\pi} d \theta_{1} d \theta_{2} \int_{-\infty}^{+\infty} d \eta_{1} d \eta_{2} d X_{1} d X_{2} w\left(X_{1}, X_{2}, \theta_{1}, \theta_{2}\right)\left|\eta_{1} \eta_{2}\right| \\
& \times \exp i\left[\eta_{1}\left(X_{1}-\hat{q_{1}} \cos \theta_{1}-\hat{p_{1}} \sin \theta_{1}\right)+\eta_{2}\left(X_{2}-\hat{q_{2}} \cos \theta_{2}-\hat{p_{2}} \sin \theta_{2}\right)\right] \geq 0 .
\end{aligned}
$$

Here $\hat{q}_{1}, \hat{q}_{2}, \hat{p}_{1}, \hat{p}_{2}$ are position and momentum operators of both quantum particles, respectively. The inequality (5) means that the eigenvalues of the operator $\hat{\rho}(1,2)$ are nonnegative numbers. Using the tomographic probability description of classical and quantum states by means of joint probability distribution one can naturally consider the hybrid situation. Let the tomogram $w\left(X_{1}, X_{2}, \theta_{1}, \theta_{2}\right)$ satisfy the following conditions. The tomogram of first particle $\Omega_{1}\left(X_{1}, \theta_{1}\right)=\int w\left(X_{1}, X_{2}, \theta_{1}, \theta_{2}\right) d X_{2}$ and tomogram of second particle $\Omega_{2}\left(X_{2}, \theta_{2}\right)=\int w\left(X_{1}, X_{2}, \theta_{1}, \theta_{2}\right) d X_{1}$ must satisfy the conditions

$$
\frac{1}{2 \pi} \int_{0}^{\pi} d \theta_{1} \int_{-\infty}^{+\infty} d \eta_{1} d X_{1} \Omega_{1}\left(X_{1}, \theta_{1}\right)\left|\eta_{1}\right| \exp i \eta_{1}\left(X_{1}-q_{1} \cos \theta_{1}-p_{1} \sin \theta_{1}\right)=f_{1}\left(q_{1}, p_{1}\right) \geq 0
$$

and

$$
\hat{\rho}(2)=\frac{1}{2 \pi} \int_{0}^{\pi} d \theta_{2} \int_{-\infty}^{+\infty} d \eta_{2} d X_{2} \Omega_{2}\left(X_{2}, \theta_{2}\right)\left|\eta_{2}\right| \exp \left[i \eta_{2}\left(X_{2}-\hat{q_{2}} \cos \theta_{2}-\hat{p_{2}} \sin \theta_{2}\right)\right] \geq 0 .
$$

These conditions mean that the integral (6) provides the probability density on phase-space of the classical first particle and the integral (7) provides density operator of the quantum state of the second particle. The joint tomogram can satisfy (3) and violate (5). Such tomogram describes classical states of two particles. Another possibility corresponds to case where the tomogram satisfies (5) and violates (3). Such tomogram describes quantum states of two particles. The joint tomogram $w\left(X_{1}, X_{2}, \theta_{1}, \theta_{2}\right)$ can describe neither classical nor quantum state of two particles violating both conditions (3) and (5). The tomograms could be used to describe hybrid system of two particles one of which is classical and another one is quantum.

\section{Correlations of quantum and classical variables}

The tomogram of hybrid system can be written in factorized form

$$
w\left(X_{1}, X_{2}, \theta_{1}, \theta_{2}\right)=\Omega\left(X_{1}, \theta_{1}\right) \Omega_{2}\left(X_{2}, \theta_{2}\right) .
$$

Here $\Omega_{1}\left(X_{1}, \theta_{1}\right)$ is classical tomogram and $\Omega_{2}\left(X_{2}, \theta_{2}\right)$ is quantum tomogram. The tomogram (8) is joint probability distribution of two random positions $X_{1}$ and $X_{2}$ of the system state which does not contain correlations of these observables. It means that behaviour of classical particle does not influence on the behaviour of the quantum particle and vice versa. But there exists the tomograms of the form

$$
w\left(X_{1}, X_{1}, \theta_{1}, \theta_{2}\right)=P \Omega_{1}\left(X_{1}, \theta_{1}\right) \Omega_{2}\left(X_{2}, \theta_{2}\right)+(1-P) \bar{\Omega}_{1}\left(X_{1}, \theta_{1}\right) \bar{\Omega}_{2}\left(X_{2}, \theta_{2}\right),
$$

where $0 \leq P \leq 1$. The tomogram (8) describes the state which is mixture (convex sum) of two joint probability distribution (5) without correlations. The mixture provides the nonzero correlation of two 
random positions. In fact the covariance of two random positions reads

$$
\begin{aligned}
& \sigma_{X_{1} X_{2}}=\left\langle X_{1} X_{2}\right\rangle-\left\langle X_{1}\right\rangle\left\langle X_{2}\right\rangle= \\
& \int w\left(X_{1}, X_{2}, \theta_{1}, \theta_{2}\right) X_{1} X_{2} d X_{1} d X_{2}-\int X_{1} w\left(X_{1}, X_{2}, \theta_{1}, \theta_{2}\right) d X_{1} d X_{2} \int X_{2} w\left(X_{1}, X_{2}, \theta_{1}, \theta_{2}\right) d X_{1} d X_{2}= \\
& \int\left(P \Omega_{1}\left(X_{1}, \theta_{1}\right) \Omega_{2}\left(X_{2}, \theta_{2}\right)+(1-P) \bar{\Omega}_{1}\left(X_{1}, \theta_{1}\right) \bar{\Omega}_{2}\left(X_{2}, \theta_{2}\right)\right) X_{1} X_{2} d X_{1} d X_{2} \\
& -\int X_{1}\left(P \Omega_{1}\left(X_{1}, \theta_{1}\right) \Omega_{2}\left(X_{2}, \theta_{2}\right)+(1-P) \bar{\Omega}_{1}\left(X_{1}, \theta_{1}\right) \bar{\Omega}_{2}\left(X_{2}, \theta_{2}\right)\right) d X_{1} d X_{2} \\
& \times \int X_{2}\left(P \Omega_{1}\left(X_{1}, \theta_{1}\right) \Omega_{2}\left(X_{2}, \theta_{2}\right)+(1-P) \bar{\Omega}_{1}\left(X_{1}, \theta_{1}\right) \bar{\Omega}_{2}\left(X_{2}, \theta_{2}\right)\right) d X_{1} d X_{2}
\end{aligned}
$$

and it is not equal to zero.

General expression for the tomogram of the state of the hybrid system, i.e. the generic convex sum of the tomograms without correlations reads

$$
w\left(X_{1}, X_{2}, \theta_{1}, \theta_{2}\right)=\sum_{k} P_{k} \Omega_{1}^{(k)}\left(X_{1}, \theta_{1}\right) \Omega_{2}^{(k)}\left(X_{2}, \theta_{2}\right)
$$

where $0 \leq P_{k} \leq 1$ and $\sum_{k} P_{k}=1$. This tomogram corresponds to the state with covariance

$$
\begin{aligned}
& \sigma_{X_{1} X_{2}}=\left\langle X_{1} X_{2}\right\rangle-\left\langle X_{1}\right\rangle\left\langle X_{2}\right\rangle= \\
& \int w\left(X_{1}, X_{2}, \theta_{1}, \theta_{2}\right) X_{1} X_{2} d X_{1} d X_{2}-\int X_{1} w\left(X_{1}, X_{2}, \theta_{1}, \theta_{2}\right) d X_{1} d X_{2} \int X_{2} w\left(X_{1}, X_{2}, \theta_{1}, \theta_{2}\right) d X_{1} d X_{2}= \\
& \int\left(\sum P_{k} \Omega_{1}^{(k)}\left(X_{1}, \theta_{1}\right) \Omega_{2}^{(k)}\left(X_{2}, \theta_{2}\right)\right) X_{1} X_{2} d X_{1} d X_{2} \\
& -\int X_{1}\left(\sum P_{k} \Omega_{1}^{(k)}\left(X_{1}, \theta_{1}, \Omega_{2}^{(k)}\left(X_{2}, \theta_{2}\right)\right) d X_{1} d X_{2}\right. \\
& \times \int X_{2}\left(\sum P_{k} \Omega_{1}^{(k)}\left(X_{1}, \theta_{1}, \Omega_{2}^{(k)}\left(X_{2}, \theta_{2}\right)\right) d X_{1} d X_{2}\right.
\end{aligned}
$$

The formula (11) can be generalized to the case of entangled states of the hybrid system. The tomogram of the entangled state by analogy of the entangled state of quantum bipartite system reads

$$
w^{e n t}\left(X_{1}, X_{2}, \theta_{1}, \theta_{2}\right)=(1+\mu) \sum_{k} P_{k} \Omega_{1}^{(k)}\left(X_{1}, \theta_{1}\right) \Omega_{2}^{(k)}\left(X_{2}, \theta_{2}\right)-\mu \sum_{k^{\prime}} P_{k^{\prime}} \Omega_{1}^{\left(k^{\prime}\right)}\left(X_{1}, \theta_{1}\right) \Omega_{2}^{\left(k^{\prime}\right)}\left(X_{2}, \theta_{2}\right)
$$

where $\mu \geq 0$.

The entangled tomogram is analogous to the form of distribution given by two numbers $z$ and $1-z$, $1 \geq z \geq 0$ which is obtained as the difference

$$
\begin{aligned}
& z=(1+\mu) x-\mu y \\
& 1-z=(1+\mu)(1-x)-\mu(1-y) \\
& \quad 1 \geq x, y \geq 0
\end{aligned}
$$

The conditions

$$
y\left(\frac{\mu}{1+\mu}\right) \leq x \leq \frac{1+y \mu}{\mu+1}
$$

guarantee that probability distribution determined by number $z$ is similar to the probability distribution for entangled states. 


\section{Evolution equation}

The tomogram of the hybrid system has to satisfy the evolution equation. We suggest the following equation which contains two contributions

$$
\begin{aligned}
& \frac{\partial}{\partial t} w\left(X_{1}, X_{2}, \theta_{1}, \theta_{2}, t\right)= \\
& {\left[\cos ^{2} \theta_{1} \frac{\partial}{\partial \theta_{1}}-\frac{1}{2} \sin 2 \theta_{1}\left\{1+X_{1} \frac{\partial}{\partial X_{1}}\right\}\right] w\left(X_{1}, X_{2}, \theta_{1}, \theta_{2}\right)} \\
& +2\left[\operatorname{Im} U_{1}\left\{q_{1} \rightarrow\left\{\sin \theta_{1} \frac{\partial}{\partial \theta_{1}}\left[\frac{\partial}{\partial X_{1}}\right]^{-1}+X_{1} \cos \theta_{1}+i \frac{\sin \theta_{1}}{2} \frac{\partial}{\partial X_{1}}\right\}\right\}\right] w\left(X_{1}, X_{2}, \theta_{1}, \theta_{2}\right) \\
& +\left[\cos ^{2} \theta_{2} \frac{\partial}{\partial \theta_{2}}-\frac{1}{2} \sin 2 \theta_{2}\left\{1+X_{2} \frac{\partial}{\partial X_{2}}\right\}\right] w\left(X_{1}, X_{2}, \theta_{1}, \theta_{2}\right) \\
& +\left[\frac{\partial}{\partial q_{2}} U_{2}\left\{q_{2} \rightarrow\left\{\sin \theta_{2} \frac{\partial}{\partial \theta_{2}}\left[\frac{\partial}{\partial X_{2}}\right]^{-1}+X_{2} \cos \theta_{2}+i \frac{\sin \theta_{2}}{2} \frac{\partial}{\partial X_{2}}\right\}\right\}\right] \sin \theta_{2} \frac{\partial}{\partial X_{2}} w\left(X_{1}, X_{2}, \theta_{1}, \theta_{2}\right) .
\end{aligned}
$$

Here $U_{1}$ and $U_{2}$ are potential energy for quantum and classical particles respectively.

For symplectic tomogram of the two-particle state $w\left(X_{1}, X_{2}, \mu_{1}, \mu_{2}, \nu_{1}, \nu_{2}, t\right)$ the evolution equation (14) has the form

$$
\begin{aligned}
& \left\{\frac{\partial}{\partial t}-\nu_{1} \frac{\partial}{\partial \mu_{1}}-\nu_{2} \frac{\partial}{\partial \mu_{2}}-\frac{1}{i}\left[U_{1}\left\{q_{1} \rightarrow\left(-\frac{\partial}{\partial \mu_{1}}\left(\frac{\partial}{\partial X_{1}}\right)^{-1}+\frac{i}{2} \nu_{1} \frac{\partial}{\partial X_{1}}\right)\right\}-c . c .\right]\right. \\
& \left.-\left[\frac{\partial U_{2}}{\partial q_{2}}\left\{q_{2} \rightarrow-\frac{\partial}{\partial \mu_{2}}\left(\frac{\partial}{\partial X_{2}}\right)^{-1}\right\}\right] \nu_{2} \frac{\partial}{\partial X_{2}}\right\} w\left(X_{1}, X_{2}, \mu_{1}, \mu_{2}, \nu_{1}, \nu_{2}, t\right)=0
\end{aligned}
$$

Both equations (14) and (15) provide the classical Liouville evolution for the classical particle tomogram and quantum von Neumann evolution for quantum particle tomogram. These two tomograms are obtained by averaging the tomogram of two particles either with respect to quantum position variable $X_{1}$ or classical position variable $X_{2}$.

The suggested equations preserve the form of tomograms given both by convex sum of tomograms without correlations and by the entangled state tomograms. On the other hand the correlation of classical and quantum observables are present for solutions of the equations.

\section{Conclusions}

To conclude we summarize the main results of the paper. We suggested to describe the states of a system containing a classical and a quantum subsystems by means of the joint tomographic probability distributions and studied the properties of such tomograms. We proposed the evolution equation for the states of such hybrid quantum-classical system. The evolution equation is shown to provide the Liouville equation for the classical subsystem tomogram and von Neumann evolution equation for quantum subsystem after corresponding averaging in the proposed equation on the quantum and classical degrees of freedom, respectively. We shown that the entanglement of the classical and quantum subsystems of the hybrid system can be formulated in terms of properties of the joint tomographic probability of the bipartite classical-quantum system. The partial case of Gaussian tomogram for two-mode electromagnetic field in the states which are generalizations of quantum separable or entangled states is worthy to study. One can check experimentally presence of classical mode by means of studying homodyne quadrature distribution 
which for classical mode can violate uncertainty relation. We point out that the suggested formalism provides extension of conventional quantum and classical mechanics.

\section{Acknowledgement}

V.N.C. and V.I.M. thank Russian Foundation for Basic Research for the support under Project Nos.0702-00598,08-02-90300, and 09-02-00142

\section{References}

[1] E. Schrödinger, Ann. Phys (Leipzig), 79, p. 489 (1926)

[2] L. D. Landau, Z. Physik, 45, p. 430 (1927)

[3] J. von Neumann, Mathematische Grundlagen der Quantenmechanik, Springer, Berlin (1932)

[4] S. Mancini, V. I. Man'ko, and P. Tombesi, Phys. Lett. A, 213, 1 (1996)

[5] A. Ibort, V. I. Man'ko, G. Marmo, A. Simoni, and F. Ventriglia, Phys. Scripta, 79, p. 065013 (2009)

[6] O. V. Man'ko, V. I. Man'ko, J. Russ. Laser Research, 18, N 2, 407 (1997)

[7] V. I. Man'ko, R. V. Mendes, Physica D, 145, p. 330 (2000)

[8] D. T. Smithey, M. Beck, M. G. Raymer, A. Faridani, Phys. Rev. Lett., 70, p. 1244 (1993)

[9] A. I. Lvovsky and M. G. Raymer, Rev. Mod. Phys., 81, p. 299 (2009)

[10] V. I. Man'ko, G. Marmo, A. Porzio, S. Solimeno, F. Ventriglia, Physica Scripta, 83045001 (2011)

[11] O. V. Man'ko, V. I. Man'ko, J. Russ. Laser Research, 25, N 2, 477 (2004)

[12] A. Peres, D. Terno, Phys. Rev. A, 63, 022101 (2001)

[13] H-T. Elze, G. Gambarotta, F. Vallone, J. Phys.: Conf.Ser., 306, 012010 (2011)

[14] E. Wigner, Phys. Rev., 40, 749 (1932)

[15] Yu. A. Korennoy, V. I. Man'ko, "Probability representation of quantum evolution and energy level equations for optical tomograms" e-Print: arXiv:1101.2537v1[quant-ph] 13 Jan. 2011

[16] G. G. Amosov, Ya. A. Korennoy, V. I. Man'ko, "Operators and their symbols in the optical probabilistic representation of quantum mechanics" e-Print: arXiv:1104.5606[quant-ph] Apr 2011. 11 pp. 